BMC

Cell Biology

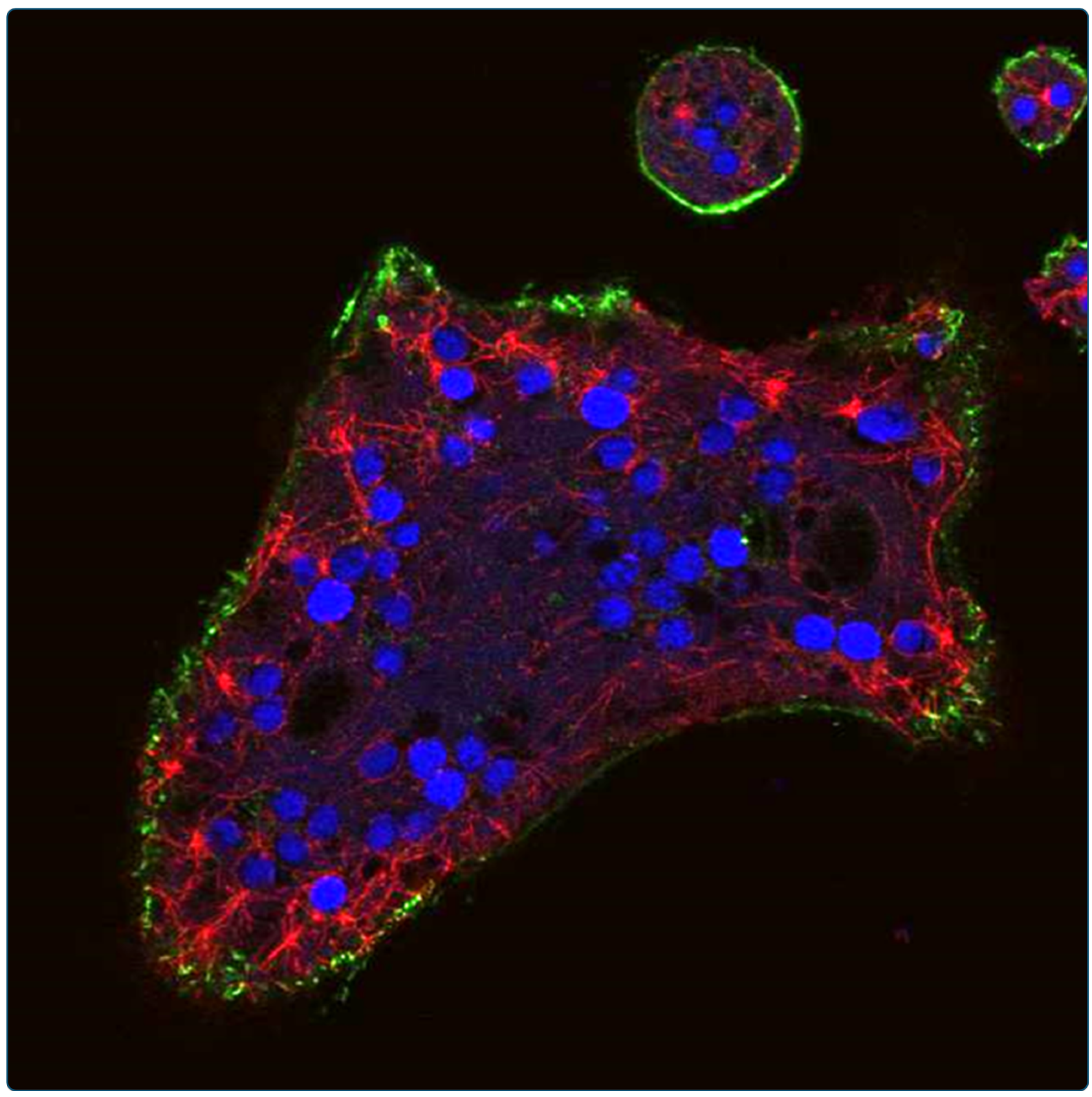

\title{
Regulation of a LATS-homolog by Ras GTPases is important for the control of cell division
}

Müller-Taubenberger et al. 


\title{
Regulation of a LATS-homolog by Ras GTPases is important for the control of cell division
}

\author{
Annette Müller-Taubenberger ${ }^{1 * \dagger}$, Peter M Kastner ${ }^{1 \dagger}$, Michael Schleicher ${ }^{1}$, Parvin Bolourani ${ }^{2}$ and Gerald Weeks ${ }^{2}$
}

\begin{abstract}
Background: Nuclear Dbf-related/large tumor suppressor (NDR/LATS) kinases have been shown recently to control pathways that regulate mitotic exit, cytokinesis, cell growth, morphological changes and apoptosis. LATS kinases are core components of the Hippo signaling cascade and important tumor suppressors controlling cell proliferation and organ size in flies and mammals, and homologs are also present in yeast and Dictyostelium discoideum. Ras proto-oncogens regulate many biological functions, including differentiation, proliferation and apoptosis. Dysfunctions of LATS kinases or Ras GTPases have been implicated in the development of a variety of cancers in humans.

Results: In this study we used the model organism Dictyostelium discoideum to analyze the functions of NdrC, a homolog of the mammalian LATS2 protein, and present a novel regulatory mechanism for this kinase. Deletion of the $n d r C$ gene caused impaired cell division and loss of centrosome integrity. A yeast two-hybrid analysis, using activated Ras proteins as bait, revealed NdrC as an interactor and identified its Ras-binding domain. Further in vitro pull-down assays showed that NdrC binds RasG and RasB, and to a lesser extent Ras C and Rap1. In cells lacking NdrC, the levels of activated RasB and RasG are up-regulated, suggesting a functional connection between RasB, RasG, and NdrC.

Conclusions: Dictyostelium discoideum NdrC is a LATS2-homologous kinase that is important for the regulation of cell division. NdrC contains a Ras-binding domain and interacts preferentially with RasB and RasG. Changed levels of both, RasB or RasG, have been shown previously to interfere with cell division. Since a defect in cell division is exhibited by NdrC-null cells, RasG-null cells, and cells overexpressing activated RasB, we propose a model for the regulation of cytokinesis by $\mathrm{NdrC}$ that involves the antagonistic control by RasB and RasG.
\end{abstract}

Keywords: Cell division, Dictyostelium discoideum, Ras GTPase, LATS kinase

\section{Background}

Nuclear Dbf-related/large tumor suppressor (NDR/LATS) kinases have been shown recently to control pathways regulating mitotic exit, cytokinesis, cell proliferation, morphological changes and apoptosis [1-3]. Work over the past decade has revealed that LATS kinases are core components of the Hippo signaling pathway that has been shown to have a tumor suppressor function and to control tissue growth in flies and mammals [4-8]. Loss of NDR/ LATS kinase activity has been related to the development of various human malignancies $[1,4,9]$.

The Hippo pathway was identified first by genetic screens in Drosophila melanogaster. The Ste20 kinase

\footnotetext{
* Correspondence: amueller@|rz.uni-muenchen.de

${ }^{\dagger}$ Equal contributors

'Anatomy III - Cell Biology, Ludwig Maximilian University of Munich,

Schillerstr. 42, 80336 Munich, Germany

Full list of author information is available at the end of the article
}

Hippo (MST1/2 in mammals) and the NDR family kinase Warts (LATS1/2 in mammals) constitute the core of the signaling cascade. The elucidation of the mechanisms that regulate the Hippo pathway and the identification of interactors contributes to our understanding how growth and organ size in flies and mammals are controlled and why misregulation leads to the formation of cancer. Recent studies in Drosophila and mammalian cells have suggested that LATS kinases are involved in the density-dependent control of cell proliferation through a cell morphologybased mechanism which is mediated by stress fibers and cooperates with a cell adhesion-based mechanism [10-12].

Homologs of the Hippo pathway components have been shown to be present in yeast [5,13], Dictyostelium discoideum [14], and Capsaspora owczarzaki [15], thus providing an opportunity for the genetic analysis of the essential and evolutionary functions of these important regulators. In particular Dictyostelium is an easily accessible eukaryotic 
model system to gain insights into a variety of basic cellular processes, including the regulatory machinery controlling cell division $[16,17]$. The LATS/NDR family of Dictyostelium consists of two LATS-related kinases, $\mathrm{NdrC}$ and $\mathrm{NdrD}$, as well as two NDR-related kinases, NdrA and NdrB $[18,19]$. In the present study, we have explored the function of $\mathrm{NdrC}$, and provide evidence that $\mathrm{NdrC}$ plays an important role in cell division. Based on the data presented, we propose that its activity is antagonistically controlled by RasB and RasG, two members of the Ras subfamily of GTPases.

\section{Results and discussion}

Identification of NdrC as a Ras GTPase interacting protein Dictyostelium discoideum NdrC (DDB0349842) belongs to the LATS/NDR kinase family, which constitutes a subgroup of AGC (protein kinase A/G/C-related) kinases $[18,20]$. The Dictyostelium LATS/NDR family consists of four kinases, two 'shorter' NDR kinases ( $\mathrm{NdrA} / \mathrm{B})$, and two 'larger' LATS/NDR-related kinases (NdrC/D) that are characterized by an extended N-terminus [19]. Similarly, the mammalian LATS/NDR kinase family is

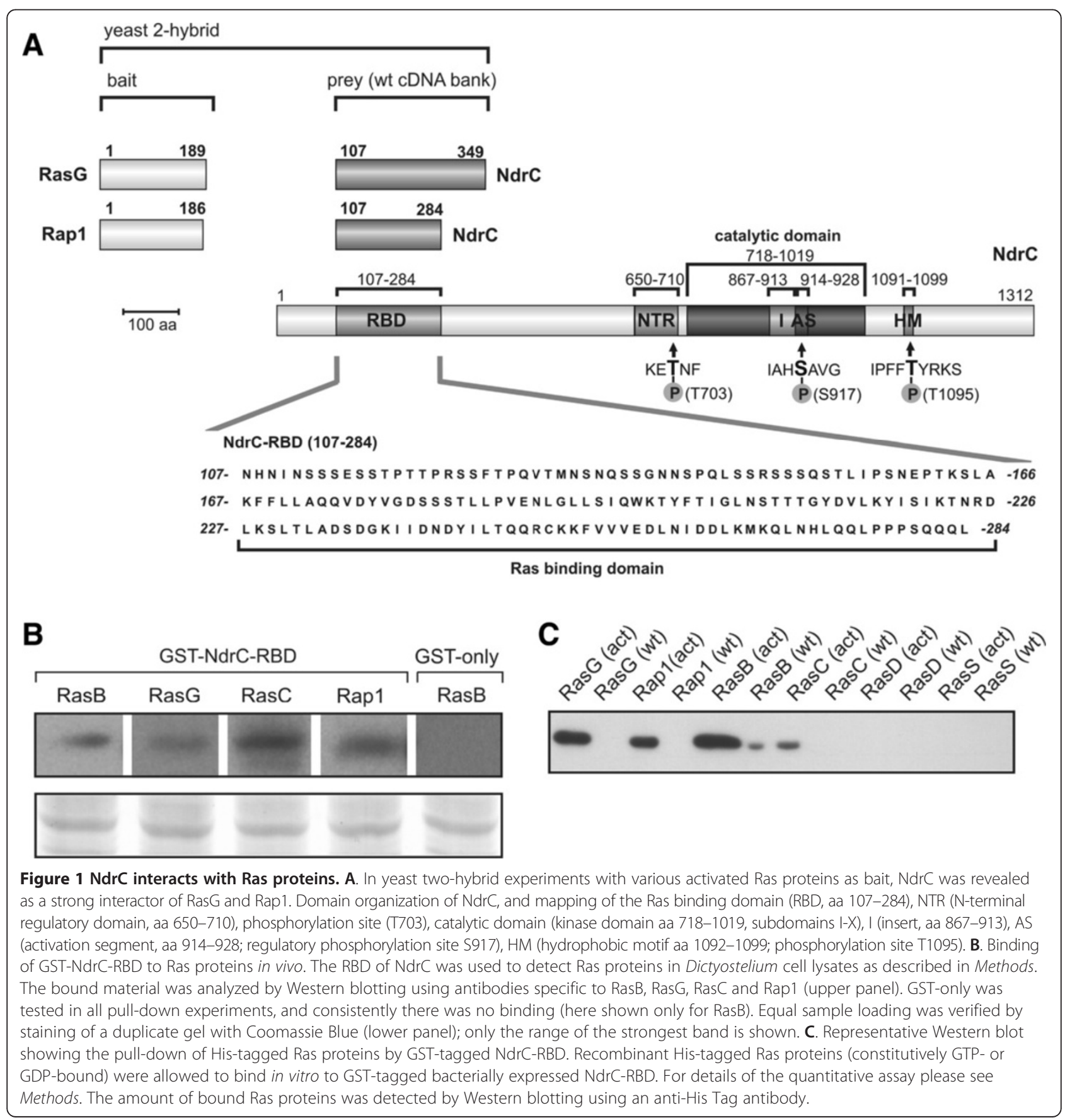


subdivided into two 'larger' LATS kinases (LATS1/2) and two 'shorter' NDR kinases (NDR1/2) (Additional file 1: Figure S1A). The Dictyostelium NdrC kinase is made up of 1,312 amino acids ( $147 \mathrm{kDa})$, and its protein sequence comprises the general features described for other LATS/NDR kinases, which include an N-terminal regulatory domain (NTR), an insert sequence (I) between subdomain VII and VIII of the catalytic domain, an activation segment (AS) as well as a conserved hydrophobic motif (HM) (Figure 1A, Additional file 1: Figure S1B).

When the Dictyostelium GTPases RasG, RasC and Rap1 were employed as bait in a yeast two-hybrid screen, $\mathrm{NdrC}$ was identified as a novel interacting protein that exhibited strong positive interactions with all three proteins. This screen also revealed that RasG and Rap1 exhibited strong positive interactions with the previously described Ras-binding domain (RBD) containing proteins PL3K and Rip3. In contrast, NdrC was the only protein that bound $\mathrm{RasC}$ in the yeast two-hybrid screen. The RBD of NdrC was localized between amino acid residues 107 and 284, the sequence that interacted with all three Ras proteins in the yeast two-hybrid assays (Figure 1A and data not shown). The minimal Ras-binding domain of $\mathrm{NdrC}$ was not defined further by additional experiments. A bioinformatics analysis did not reveal evidence of a RBD sequence in this region, but this is not surprising since the RBD sequences of a number of other RBD proteins were not detected by bioinformatic analyses [21].

Ras protein binding to $\mathrm{NdrC}$ was confirmed by pulldown assays using the identified RBD of $\mathrm{NdrC}$ tagged to GST and Dictyostelium lysates (Figure 1B). Bound Ras proteins were detected by Western blot analysis using specific anti-Ras polyclonal antibodies. The experiment revealed that the NdrC-RBD interacted not only with RasG, RasC and Rap1, but also with RasB (Figure 1B). This result was confirmed and extended by pull-down experiments using GST-tagged NdrC-RBD in combination with His-tagged Ras subfamily proteins that allowed a more quantifiable comparison of the binding. These interaction studies showed that $\mathrm{NdrC}$ bound to the activated Ras proteins but not to the wild type (Figure 1C), although the wild-type proteins did bind in the presence of GTPYS (data not shown). NdrC bound best to activated RasG and RasB, with lower levels of binding to activated Rap1 and RasC (Figure 1C). NdrC did not interact with either RasD or RasS (Figure 1C).

\section{ndrC-null cells exhibit a severe defect in cytokinesis and aberrant numbers of centrosomes}

To investigate the cellular function of $\mathrm{NdrC}$, a knockout strain of the $n d r C$ gene was generated by homologous recombination using a disruption construct carrying an inverted blasticidin-S resistance cassette within the partial $n d r C$ gene sequence (Figure 2A). The most striking phenotype of the $n d r C$-null cells when compared to the wild type was that the majority of mutant cells were larger and multinucleated (Figure 2B). When wild-type and $n d r C$-null cells were fixed and stained with TO-PRO-3 to visualize the nuclei, and counterstained for actin using Alexa Fluor 488-coupled phalloidin, the increased number of nuclei in the mutant was clearly revealed (Figure $2 \mathrm{C}, \mathrm{D}$ ), and this increase was quantified by nuclei counts of fixed, DAPIstained cell preparations (Figure 2E). The percentage of mono-nucleated cells in wild type was $95 \%$ compared to only $29 \%$ in $n d r C$-null cells (Figure $2 \mathrm{E}$ ). The number of nuclei per cell in the $n d r C$-nulls peaked at 1,2, 4 and 8 indicating concerted mitosis (Figure 2E).

An analysis of living cells lacking $\mathrm{NdrC}$ revealed that they were able to perform mitosis, but an impairment of cell division resulted in the formation of multinuclear cells. Frequently, putative daughter cells were still connected by thin cellular bridges suggesting division by cytofission rather than mitosis-coupled cytokinesis (Figure 2B, right). The generation times, measured for $n d r C$-null and wild-type cells were almost identical, regardless of whether cells were grown in shaking culture in rich axenic medium or on a solid substrate with bacterial lawns (Additional file 2: Figure S2). Since $n d r C$-null cells are considerably larger than wild-type cells, their cell masses increase at considerably faster rates indicating an additional defect in the control of cell growth.

Inspection of multinucleated $n d r C$-null cells, fixed and stained with TO-PRO-3 for visualization of nuclei and immunostained with anti- $\alpha$-tubulin antibodies to visualize microtubules and centrosomes, revealed a high percentage of mutant cells carrying increased numbers of centrosomes (Figure 3). Frequently, the supernumerous centrosomes were not connected to the nuclei (Figure 3B). To quantify the disruption of centrosome integrity, the ratio of centrosomes visualized by anti- $\alpha$-tubulin staining to nuclei visualized by DAPI staining, was determined for wildtype and $n d r C$-null cells (Figure 3D). About one third (31\%) of the $n d r C$-null cells had more than one centrosome per nucleus, compared to only $0.6 \%$ in wild-type cells. $n d r C$-null cells with evenly distributed centrosomes underwent mitosis in a synchronized manner (concerted mitosis) (Figure 3E), but although the increased ratio of centrosomes per nuclei did not affect the ability of cells to perform concerted mitosis, the unattached surplus centrosomes were not capable of forming mitotic spindles (Figure 3F).

Aberrant numbers of centrosomes have also been observed for knockout strains of mouse LATS2 $[22,23]$ and human NDR1/2 [3,24]. The cell division defect in mammalian cells has been attributed to a disruption of the signaling cascade controlling cytokinesis on the one hand [25], and a control of nuclear division on the other 

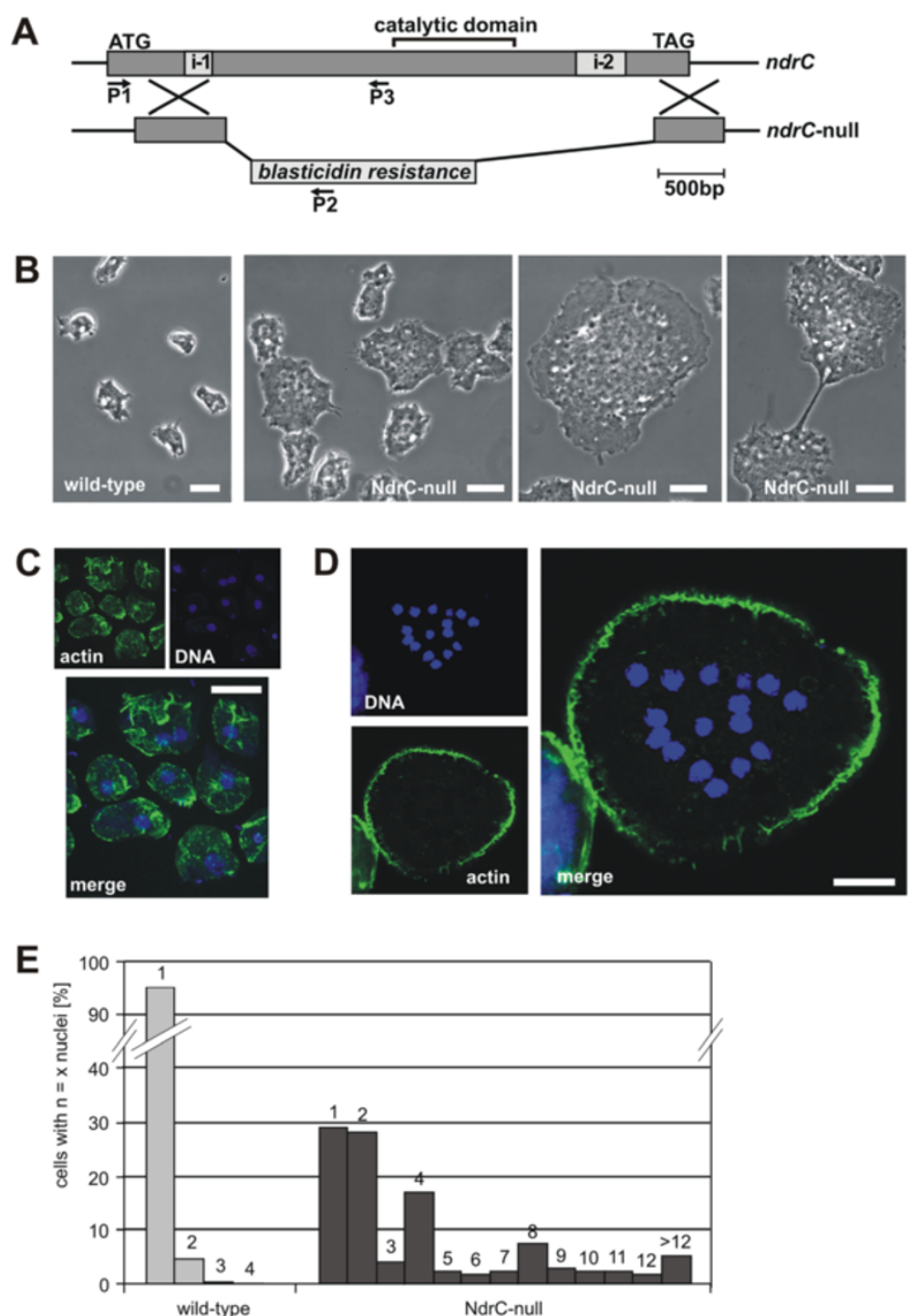

Figure 2 NdrC-null cells are impaired in cell division. A. Gene replacement of ndrC (DDB0219984) by a blasticidin-S resistance cassette. P1-P2 and P1-P3 indicate the primer combinations that were used initially to identify gene knockouts by PCR. B. Live-cell microscopy of wild-type (left) and ndrC-null cells. ndrC-null cells are much larger than wild-type cells, and often divide by traction-mediated cytofission (as shown in the right image). C. Fixed wild-type cells stained with TRITC-phalloidin for actin and TO-PRO-3 for DNA. D. Fixed ndrC-null cell stained with TRITC-phalloidin for actin and TO-PRO-3 for DNA. E. Histogram showing the percentage of cells carrying the indicated numbers of nuclei in wild-type and ndrC-null mutants. Cells were grown in Petri dishes, fixed, stained, and for each strain the nuclei of $>500$ cells were counted. The counting was repeated in an independent experiment with almost identical results. Bars, $10 \mu \mathrm{m}$.

[26]. Human LATS1 was also reported to act as mitotic exit network kinase [27], responsible for regulation of the G2/M-arrest [28]. In addition, the Drosophila LATShomolog Warts regulates mitotic progression [29]. However, despite these clear indications for a role of NDR/ LATS kinases in regulation of the cell cycle and/or cell division, the underlying mechanisms are largely unknown.

\section{Subcellular localization of $\mathrm{NdrC}$}

Gene expression profiling data indicated that $\mathrm{NdrC}$ is expressed at very low levels throughout the developmental cycle of Dictyostelium (http://dictyexpress.biolab.si) [30]. In order to specify the cellular localization of $\mathrm{NdrC}$, polyclonal antibodies were raised against $\mathrm{NdrC}$. Immunolabeling experiments with fixed Dictyostelium cells indicated that $\mathrm{NdrC}$ is preferentially enriched at the centrosome (Figure 4A). This localization was confirmed by co-staining with a monoclonal antibody directed against a genuine centrosomal component, the corona protein CP224 [31]. The centrosomal localization of $\mathrm{NdrC}$ was detectable only in a subfraction of cells, suggesting a cell cycle-dependent enrichment. Expression of full-length 

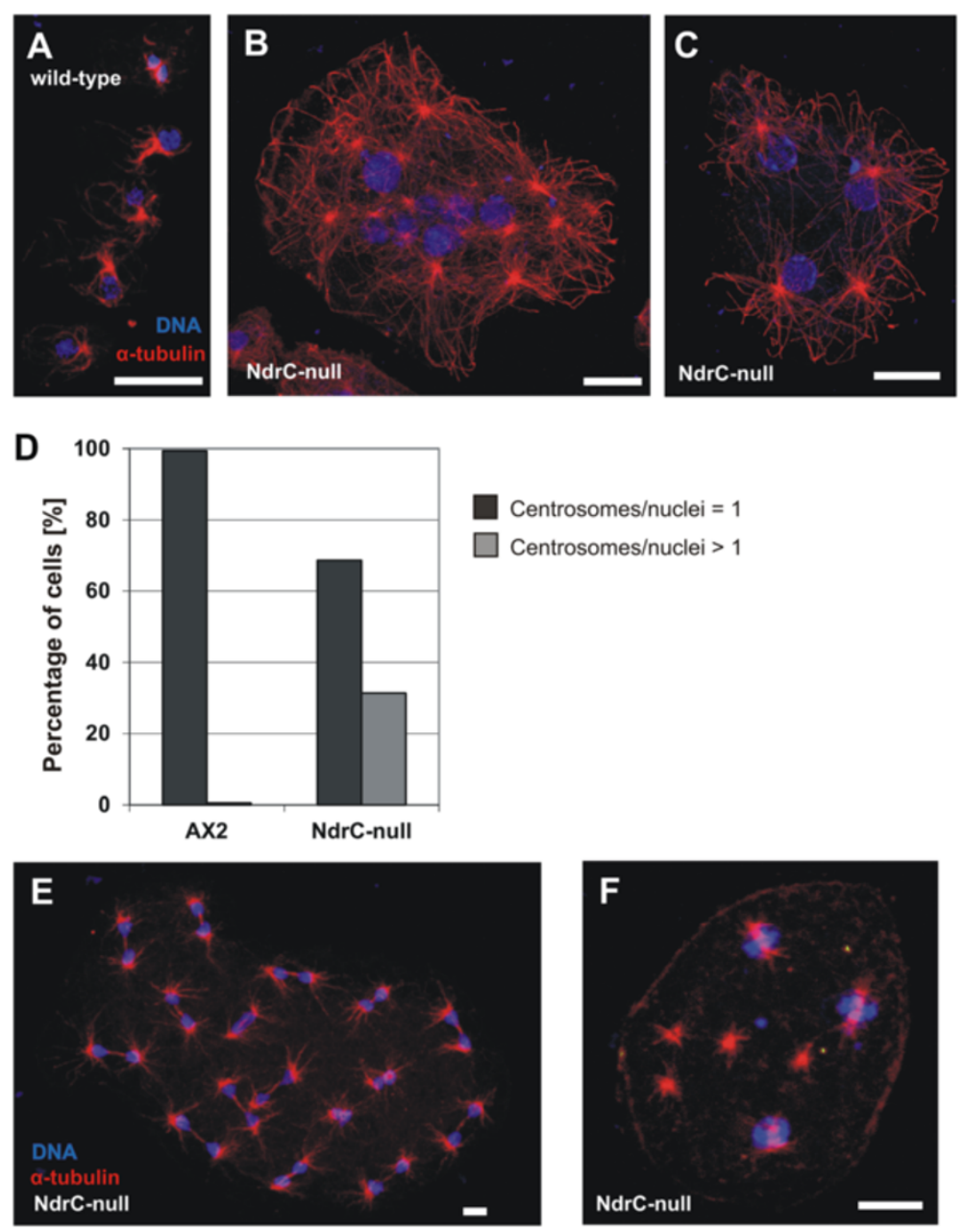

Figure 3 Cells lacking NdrC show centrosomal aberrations. A. In comparison to wild-type, B, C multinucleated ndrC-null cells frequently show supernumerous centrosomes. D. Histogram depicting the percentage of normal (centrosomes/nuclei $=1$ ) and aberrant (centrosomes/nuclei $>1$ ) centrosomes in wild-type and ndrC-null cells. E, F. Visualization of mitotic spindles in fixed ndrC-null cells by immunolabeling with anti-a-tubulin antibodies, and staining of DNA with TO-PRO-3. Spindle formation occurs synchronously in multinucleate cells (E), as well as in multinucleate cells with supernumerous centrosomes (F). Bars $5 \mu \mathrm{m}$ in (A), and, $10 \mu \mathrm{m}$ in (B), (C), (E), and (F).

$\mathrm{NdrC}$ with a N-terminal GFP-tag confirmed the localization at the centrosome. $\mathrm{NdrC}$ had not been detected in a previous proteome screen of the Dictyostelium centrosome [32], but was found to co-purify with centrosomes that were isolated from Dictyostelium wild-type and cells expressing GFP-NdrC (Additional file 3: Figure S3).

Over-expression of a fusion protein comprising GFP and the N-terminal 300 amino acids of $\mathrm{NdrC}$ containing the RBD domain, showed a very similar localization as that reported previously for the Ras-binding domain of human Raf1 that relocates upon stimulation to active zones of the cell cortex [33,34] (Figure 4B). This finding indicates a preferential binding of RBD of $\mathrm{NdrC}$ to membrane-associated Ras proteins. A GFP-fusion protein comprising the catalytic domain and the C-terminus of $\mathrm{NdrC}$ (amino acid residues 435 to 1312) localized to the cytoplasm and showed no specific enrichment (Additional file 4: Figure S4).
Putative control of NdrC by Ras GTPase family members

We have shown previously that rasG-null cells or cells overexpressing RasB-(G12T) exhibit a multinucleated cell phenotype that is similar to the one exhibited by the $n d r C$ null cells [35,36]. In addition, severe defects in cytokinesis in cells overexpressing the exchange factor RasGEF-Q, which acts specifically upstream of RasB have been described [37]. Given that NdrC contains a RBD, it is conceivable that RasB or RasG exert regulatory functions on $\mathrm{NdrC}$.

To further investigate the possible roles of RasG or RasB in NdrC function, we determined the subcellular localization of RasG and RasB. Wild-type cells overexpressing GFP-tagged RasB or RasG were normal in respect to cell size and nuclei number, and the GFP-tagged proteins predominately localized to the cortex (Figure 4C, D). The GFP-tagged activated forms of the proteins, RasB(G12T) and RasG-(G12T), also localized predominately to 

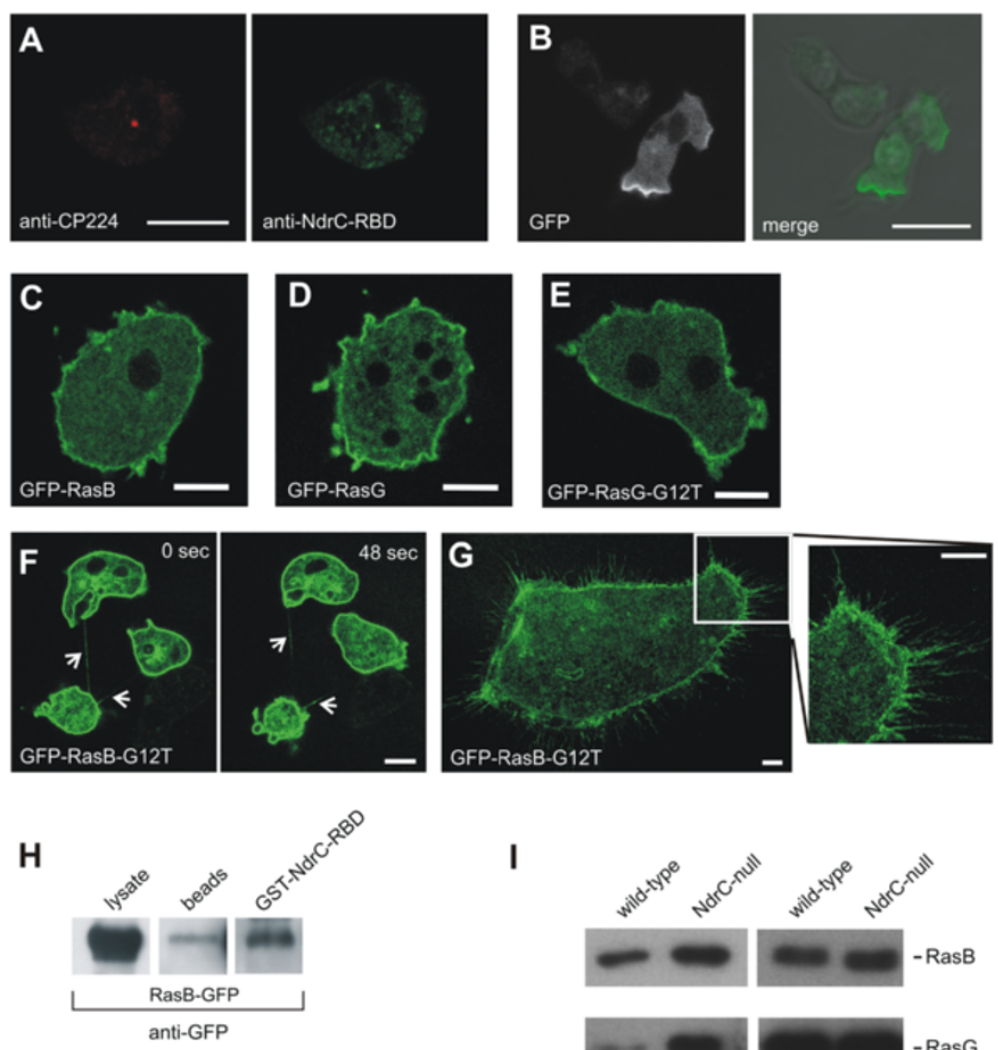

I
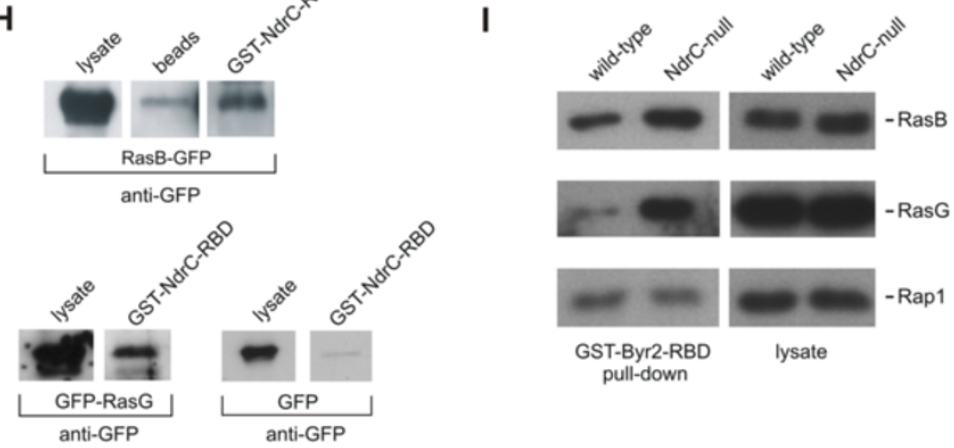

Figure 4 Localization and interaction of NdrC with Ras proteins. A. NdrC localizes to the centrosome. Fixed Dictyostelium cells were immunolabeled with centrosome-specific monoclonal anti-CP224 antibodies, and polyclonal anti-NdrC antibodies. Primary antibodies were detected with anti-mouse Alexa Fluor-568 and anti-rabbit Alexa Fluor-488 antibodies. In NdrC-null cells, no staining with anti-NdrC antibodies was detected. Bar, $10 \mu \mathrm{m}$. B. Live-cell imaging of a Dictyostelium cell expressing GFP-NdrC-RBD. Left image, GFP-NdrC-RBD signal; right image, merge. Bar, $10 \mu \mathrm{m}$. C. Live-cell imaging of GFP-RasB expressing wild-type cells shows localization of GFP-RasB to the cell cortex. D. GFP-RasG localization to the cortex of wild-type cells, shown by live cell microscopy. E. GFP-RasG(G12T) localizes to the cortex and filopodia of wild-type cells. F. Cytofission of GFP-RasB(G12T) expressing wild-type cell. G. GFP-RasB(G12T) expressed in wild-type cells localizes to the cortex and filopodia (image enlargement on the right), and results in enlarged multinucleate cells. Bars in (C)-(G), 5 Hm. H. GST-NdrC-RBD pull-down of RasB tagged to GFP shows interaction and thereby activity of the GFP-tagged Ras-GTPase. GFP-tagged RasG overexpressed in wild-type cells interacts with the GST-NdrC-RBD. The levels of bound RasG were detected by Western blotting using anti-GFP antibodies. I. Levels of activated Ras proteins in wild-type compared to ndrC-null cells. Total cell extracts from wild-type or ndrC-null cells were bound to GST-Byr2-RBD as described in Methods. The amount of activated Ras proteins pulled down or of total Ras protein in the lysate was determined by Western blotting using specific polyclonal antibodies against RasB, RasG, and Rap1. The data shown is for a single experiment, but similar results were obtained in two other experiments.

the cortex (Figure 4E-G). However, whereas RasG-(G12T) overexpression did not affect the cell phenotype (Figure 4E), overexpression of RasB-(G12T) caused disturbed cytokinesis characterized by an impairment of cells to sever the connection between daughter cells (Figure 4F). This resulted in enlarged, multinucleated cells (Figure 4F), a defect similar to that described previously for cells expressing an untagged RasB-(G12T) [35].

Both, GFP-tagged RasB and GFP-RasG bound to the GST-NdrC-RBD, as shown by in vitro pull-down assays of wild-type AX2 cells expressing GFP-tagged Ras proteins (Figure 4H). Although NdrC also binds RasC and Rap1, cell division is not affected by either the loss or upregulation of Rap1 [38], or by the loss or up-regulation of $\mathrm{RasC}$ [39]. Thus the significance of the binding of Rap1 and RasC to NdrC remains to be established.

Further evidence for a possible functional connection between the Ras-GTPases and NdrC was obtained by measuring the levels of activated Ras protein in the $n d r C$-null cells by Western blot analysis of GST-Byr2- 
RBD pull-downs using specific antibodies for the Ras family members. Levels of activated RasB and RasG were increased in the absence of $\mathrm{NdrC}$ compared to wild-type cells, indicating that $\mathrm{NdrC}$ acts as a negative regulator for activation of RasG and RasB (Figure 4I). In contrast, the levels of activated Rap1 and the total levels of all the Ras GTPases were found to be identical in lysates of wild-type and $\mathrm{NdrC}$-null cells (Figure 4I). The mechanism involved in the feedback inhibition of RasG and RasB activation by $\mathrm{NdrC}$, presumably at the level of RasGEF activity, is not known at this time, but it appears to be a general mechanism for downstream Ras effectors in Dictyostelium, since similar results have been observed for pik null and ripA null cells.

The findings that an absence of RasG and the overexpression of activated RasB both result in a multinucleate phenotype suggest the possibility that RasB and RasG play antagonistic roles in regulating $\mathrm{NdrC}$ during cytokinesis (Figure 5). The multinucleate phenotype of rasG-null cells [36] is consistent with a role for RasG in activating $\mathrm{NdrC}$, whereas the multinucleate phenotype generated by over-expression of either activated RasB [35], or the RasBspecific exchange factor GefQ [37], indicates that RasB is a negative regulator of cytokinesis and cell division.

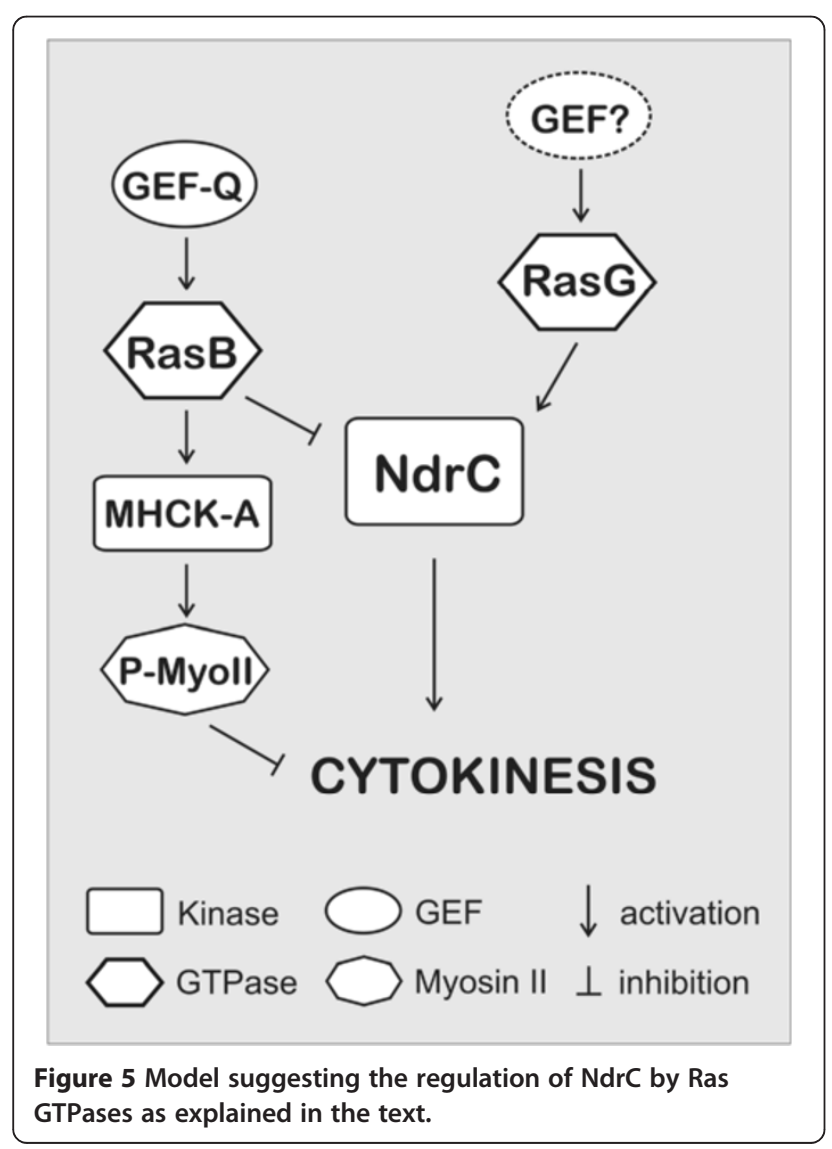

The regulation of NdrC by RasG and RasB implies that these Ras proteins would also be localized at the centrosome or in the nucleus. When cells were labeled with GFP-tagged Ras proteins, we find wild-type RasB and RasG, as well as constitutively active forms of the two proteins, are localized predominantly at the plasma membrane. This result contrasts somewhat with a previous study, using antibody staining, that revealed RasB clearly localized at the nucleus [35]. When we stained cells with an antibody highly specific to RasG, the majority of the stain was associated with the cell cortex, but some stain was also detected in the cytoplasm and in the nucleus (Additional file 5: Figure S5). These results imply that only a small proportion of the cellular RasG and RasB is localized in the nucleus, and are consistent with RasG and RasB both having other functions in the cell. In fact, RasG is not only involved in cytokinesis, but also in folate chemotaxis, in maintenance of cell shape, and in cell motility [40]. In addition, it has been shown recently that RasG, acting through PI3K, is important for pinocytosis [41]. Evidence for other specific functions for RasB is less direct, but gefQ-null cells exhibit myosin-II over-assembly and defects in polarity [37], implying a possible role for RasB in these functions, and rasB appears to be an essential gene [35], suggesting an additional vital function for RasB.

\section{Conclusions}

We present evidence for a novel mechanism for the regulation of cell division in Dictyostelium, involving the LATS2-homolog NdrC and members of the Ras subfamily of GTPases, RasB and RasG. We propose an antagonistic regulation, whereby the $\mathrm{NdrC}$ is activated by $\mathrm{RasG}$ and inactivated by RasB. The previously described interaction between the Schizosaccharomyces pombe Cdc42, a small GTPase, and Orb6, a member of the LATS/NDR group of kinases [42] suggests the possible generality of this type of mechanism, and it will be important to investigate whether the mammalian LATS kinases are also regulated by small GTPases during cell division.

\section{Methods}

\section{Cell culture, gene replacement and transformation of Dictyostelium}

Cells of the Dictyostelium discoideum wild-type strain AX2-214, or mutant cells derived from it, were cultivated at $21^{\circ} \mathrm{C}$ in nutrient HL5 medium (Formedium), either in shaken culture at $150 \mathrm{rpm}$ or in Petri dishes without shaking. To induce starvation, cells were washed twice in $17 \mathrm{mM}$ Soerensen's phosphate buffer (PB), $\mathrm{pH}$ 6.0, and shaken at a density of $10^{7}$ cells per $\mathrm{ml}$ in the buffer.

To generate $n d r C$-null mutants, wild-type AX2 cells were transformed with the gene replacement construct (Figure 2A) by electroporation using a Bio-Rad gene pulser 
at $0.8-0.9 \mathrm{kV}$ and $3 \mu \mathrm{F}$, and 4-mm cuvettes. Independent transformants were selected by addition of $7.5 \mu \mathrm{g} / \mathrm{ml}$ of Blasticidin-S (ICN Biomedicals Inc.). Transformants were cloned by spreader dilutions on lawns of non-pathogenic Klebsiella aerogenes. Three independent NdrC-knockout clones were identified by testing genomic DNA for insertion of the resistance cassette into the $n d r C$ gene by PCR.

For expression of fluorescently tagged Ras GTPases, AX2 wild-type and $\mathrm{NdrC}$-null cells were transformed by electroporation with $\mathrm{pDEX}$ derived plasmids enabling the expression of GFP-RasB, GFP-RasB-G12T, GFP-RasG and GFP-RasG-G12T. Transformants were selected by addition of $10 \mu \mathrm{g} / \mathrm{ml}$ of G418 (Sigma-Aldrich) or $7.5 \mu \mathrm{g} / \mathrm{ml}$ Blasticidin-S (ICN Biomedicals Inc.), and cloned.

\section{Live-cell microscopy}

Cells were transferred into an open chamber and washed twice with Soerensen phosphate buffer. Confocal images were taken using an inverted LSM 510 Meta confocal microscope (Zeiss) equipped with a $63 \times$ Neofluar 1.4 or a $100 \times$ Neofluar 1.3 oil immersion objective. For excitation, the 488-nm argon ion laser line and the 543-nm as well as the 633-nm helium neon laser lines were used, and emission was collected using 505-530 nm, 585-615 nm band-pass or a $650 \mathrm{~nm}$ long-pass filter.

\section{Immunofluorescence}

For immunolabeling, AX2 wild-type or $n d r C$-null cells, settled onto glass coverslips, were fixed with a mixture of $15 \%(\mathrm{v} / \mathrm{v})$ of saturated picric acid and $2 \%(\mathrm{w} / \mathrm{v})$ paraformaldehyde in $10 \mathrm{mM}$ Pipes- $\mathrm{HCl}, \mathrm{pH} 6.0$, at room temperature for $20 \mathrm{~min}$, and post-fixed with $70 \%$ ethanol for $10 \mathrm{~min}$. $\alpha$-tubulin was detected using monoclonal rat antibodies (YL1/2) [43] and Alexa Fluor 568-conjugated goat anti-rat IgG (Invitrogen). For visualization of filamentous actin, cells were stained with Alexa Fluor 488phalloidin (Molecular Probes). DNA was visualized either with TO-PRO-3 or with DAPI.

Polyclonal antibodies were generated against GST-tagged $\mathrm{NdrC}$-RBD. The sequence encoding amino acid residues 1 to 299 of $\mathrm{NdrC}$ was cloned into the bacterial expression vector pGEX-6P1 (GE Healthcare). Purification of the GSTfusion protein was performed using standard procedures, and the recombinant protein was used to immunize a female white New Zealand rabbit together with the adjuvant Gerbu100 (Gerbu Biochemicals).

\section{GST-RBD pull-down assay of endogenous Ras proteins}

The binding of endogenous activated Ras proteins to the GST-Ras Binding Domain (GST-RBD) of Dictyostelium $\mathrm{NdrC}$ was determined as described previously [39]. $350 \mu \mathrm{l}$ of Dictyostelium wild-type AX2 or $n d r C$-null cell suspension $\left(5 \times 10^{7}\right.$ cells $\left./ \mathrm{ml}\right)$ were lysed by mixing with an equal volume of $2 \times$ lysis buffer. $400 \mu \mathrm{g}$ of protein lysate were incubated with $100 \mu \mathrm{g}$ of GST-RBD and glutathione sepharose beads (GE Healthcare) at $4^{\circ} \mathrm{C}$ for 1 hour. $50 \mu \mathrm{l}$ of $1 \times$ SDS gel loading buffer was added to the pelleted beads, and the suspension was boiled for $5 \mathrm{~min}$. Samples were subjected to SDS-PAGE, and Western blots were probed with polyclonal antibodies directed against RasB, RasG, RasC, or Rap1. Equal sample loading was verified by staining of a duplicate gel with Coomassie Blue.

\section{Interaction of His-tagged Ras and GST-NdrC}

To produce 6xHis-tagged Ras-GTPases, wild-type RasG, RasC , RasB, RasS, Rap1 and RasD, and the constitutively activated forms of RasG (G12T), RasC (G13T), RasB (G15T), RasS (G12T), Rap1 (Q65E) and RasD (G12T) were cloned into pET-21a (Novagen) [41]. To express GST-NdrC, a fragment encoding amino acid residues 1-299 of NdrC was cloned into pGEX6P-1. Recombinant proteins were expressed in Escherichia coli BL21 (DE3) codonplus-RIL (Stratagene), and protein concentrations were determined using the DC Protein Assay (Bio-Rad). His-Ras/RBD-NdrC-GST interaction was examined as described previously [41]. In brief, $400 \mu \mathrm{g}$ of purified His-tagged Ras protein were incubated with $100 \mu \mathrm{g}$ of GST-RBD on glutathione sepharose beads and tumbled in binding buffer at $4^{\circ} \mathrm{C}$ overnight. Beads were washed three times and analyzed by SDS-PAGE, and Western blots were probed with anti-His tag monoclonal antibody (Santa Cruz Biotechnology).

\section{Additional files}

\begin{abstract}
Additional file 1: Figure S1. Comparison of NdrC to other LATS/NDR kinases and LATS/NDR kinase sequence signatures of Dictyostelium $\mathrm{NdrC}$. A. Sequence identities of the catalytic domain of $D$. discoideum $\mathrm{NdrC}$ with the catalytic domains of $\mathrm{D}$. discoideum $\mathrm{NdrD}, \mathrm{Ndr} \mathrm{A}$ and $\mathrm{NdrB}$, in comparison to Homo sapiens NDR1 and 2, and LATS1 and 2, and the Drosophila melanogaster LATS-homolog Warts. Numbers indicate per cent identity within the catalytic domains compared to $\mathrm{NdrC}$ catalytic domain determined by BLASTp. B. Sequence signatures of Dictyostelium NdrC. The NTR region (amino acid residues 650 to 710) carries a conserved phosphorylation site at threonine 703. The catalytic domain (subdomains I-X; amino acid residues 718 to 1019) contains an AGC-kinase specific insert (l; amino acid residues 867 to 913) as well as an adjacent activation segment (AS; amino acid residues 914 to 928) containing a conserved regulatory phosphorylation site at serine 917 . The conserved hydrophobic motif (HM; amino acid residues 1091 to 1099) corresponds to the consensus sequence $F_{-} X_{-} X_{-} Y / F_{-} T_{-} Y / F_{-}$K/R carrying a putative phosphorylation site at threonine 1095 [1].
\end{abstract}

Additional file 2: Figure S2. Growth rates of ndrC-null cells compared to wild-type. A. Growth rates of $n d r C$-null cells compared to wild-type cells in rich medium under shaking conditions. B. Growth of wild-type and ndrC-null cells on bacterial lawns of non-pathogenic K. aerogenes on agar plates.

Additional file 3: Figure S3. $\mathrm{NdrC}$ co-purifies with centrosomes. Centrosomes were isolated from cells expressing GFP-NdrC by purification of nuclei followed by pyrophosphate treatment and sucrose density centrifugation. The nuclei fraction with the associated centrosomes was

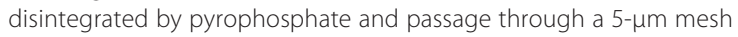
polycarbonate filter. Centrosomes were isolated via two consecutive sucrose step gradients of $80 \%$ and $50 \%$, followed by $80 \%, 70 \%, 55 \%$ and $50 \%$ 
steps in SW-40 tubes (Beckman) centrifuged at 55,000 $\times \mathrm{g}$ for $1 \mathrm{~h}$ at $4^{\circ} \mathrm{C}$. Immunostaining of methanol-fixed centrosomes was performed with monoclonal anti-CP224 antibodies [31]. The primary antibodies were visualized with Alexa Fluor-568 anti-mouse lgG (Invitrogen). Centrosomes labeled by anti-CP224 antibodies are red, those containing GFP-NdrC are green, and those containing both labels are yellow. Very similar results were obtained with centrosomes isolated from wild-type cells and immunostaining with polyclonal anti-NdrC-RBD antibodies and Alexa Fluor-488 anti-rabbit lgG.

Additional file 4: Figure S4. Localization of GFP-NdrC(435-1312). A. Scheme of the GFP-tagged NdrC (435-1312) construct. B. Live-cell imaging of a Dictyostelium wild-type cell expressing GFP-NdrC(435-1312). Bar, 5 um.

Additional file 5: Figure S5. Immunolocalization of RasG in wild-type cells. Wild-type cells were fixed and immunostained with polyclonal antibodies directed specifically against RasG. Primary antibodies were detected with Alexa Fluor-488 anti-rabbit lgG (green). Nuclei were visualized by staining with DAPI (blue). Bar, $5 \mu \mathrm{m}$.

\section{Abbreviations}

aa: Amino acids; GST: Glutathione-S-transferase.

\section{Competing interests}

The authors declare that they have no competing interests.

\section{Authors' contributions}

AMT and GW designed and supervised research; AMT, PMK and PB performed research; MS advised experiments and contributed financially; AMT, PMK, PB and GW analyzed data; AMT and GW wrote the manuscript. All authors read and approved the final manuscript.

\section{Acknowledgements}

The excellent technical assistance of Thi-Hieu Ho, Marlis Fürbringer and Gudrun Trommler is gratefully acknowledged. We thank Ralph Gräf (Potsdam) for the anti-CP224 antibodies, and Arjan Kortholt (Groningen) for providing the full-length GFP-NdrC construct. This work was supported by grants from the Deutsche Forschungsgemeinschaft to AMT and MS (SFB 914, TP7), the Canadian Institute of Health Research to GW, and the Elitenetzwerk Bayern and Universität Bayern e.V. to PMK.

\section{Author details}

'Anatomy III - Cell Biology, Ludwig Maximilian University of Munich, Schillerstr. 42, 80336 Munich, Germany. ${ }^{2}$ Department of Microbiology and Immunology, University of British Columbia, Vancouver, BC V6T 1Z3, Canada.

Received: 31 January 2014 Accepted: 25 June 2014

Published: 1 July 2014

\section{References}

1. Hergovich A, Stegert MR, Schmitz D, Hemmings BA: NDR kinases regulate essential cell processes from yeast to humans. Nat Rev Mol Cell Biol 2006, 7(4):253-264.

2. Hergovich A: Regulation and functions of mammalian LATS/NDR kinases: looking beyond canonical Hippo signalling. Cell Biosci 2013, 3(1):32.

3. Hergovich A, Cornils H, Hemmings BA: Mammalian NDR protein kinases: from regulation to a role in centrosome duplication. Biochim Biophys Acta 2008, 1784(1):3-15.

4. Hergovich A, Hemmings BA: Mammalian NDR/LATS protein kinases in hippo tumor suppressor signaling. Biofactors 2009, 35(4):338-345.

5. Hergovich A, Hemmings BA: Hippo signalling in the G2/M cell cycle phase: lessons learned from the yeast MEN and SIN pathways. Semin Cell Dev Biol 2012, 23(7):794-802.

6. Avruch J, Zhou D, Fitamant J, Bardeesy N, Mou F, Barrufet LR: Protein kinases of the Hippo pathway: regulation and substrates. Semin Cell Dev Biol 2012, 23(7):770-784.

7. Saucedo LJ, Edgar BA: Filling out the Hippo pathway. Nat Rev Mol Cell Biol 2007, 8(8):613-621.

8. Harvey K, Tapon N: The Salvador-Warts-Hippo pathway - an emerging tumour-suppressor network. Nat Rev Cancer 2007, 7(3):182-191.

9. Zeng $Q$, Hong W: The emerging role of the hippo pathway in cell contact inhibition, organ size control, and cancer development in mammals. Cancer Cell 2008, 13(3):188-192.
10. Fernandez BG, Gaspar P, Bras-Pereira C, Jezowska B, Rebelo SR, Janody F: Actin-Capping Protein and the Hippo pathway regulate F-actin and tissue growth in Drosophila. Development 2011, 138(11):2337-2346.

11. Sansores-Garcia L, Bossuyt W, Wada K, Yonemura S, Tao C, Sasaki H, Halder G: Modulating F-actin organization induces organ growth by affecting the Hippo pathway. EMBO J 2011, 30(12):2325-2335.

12. Wada K, Itoga K, Okano T, Yonemura S, Sasaki H: Hippo pathway regulation by cell morphology and stress fibers. Development 2011, 138(18):3907-3914.

13. Rock JM, Lim D, Stach L, Ogrodowicz RW, Keck JM, Jones MH, Wong CC, Yates JR 3rd, Winey M, Smerdon SJ, Yaffe MB, Amon A: Activation of the yeast Hippo pathway by phosphorylation-dependent assembly of signaling complexes. Science 2013, 340(6134):871-875.

14. Artemenko Y, Batsios P, Borleis J, Gagnon Z, Lee J, Rohlfs M, Sanseau D, Willard SS, Schleicher M, Devreotes PN: Tumor suppressor Hippo/MST1 kinase mediates chemotaxis by regulating spreading and adhesion. Proc Natl Acad Sci U S A 2012, 109(34):13632-13637.

15. Sebe-Pedros A, Zheng Y, Ruiz-Trillo I, Pan D: Premetazoan origin of the hippo signaling pathway. Cell Rep 2012, 1(1):13-20.

16. Müller-Taubenberger A, Kortholt A, Eichinger L: Simple system-substantial share: the use of Dictyostelium in cell biology and molecular medicine. Eur J Cell Biol 2013, 92(2):45-53.

17. Williams RS, Boeckeler K, Gräf R, Müller-Taubenberger A, Li Z, Isberg RR, Wessels D, Soll DR, Alexander $H$, Alexander S: Towards a molecular understanding of human diseases using Dictyostelium discoideum. Trends Mol Med 2006, 12(9):415-424.

18. Goldberg JM, Manning G, Liu A, Fey P, Pilcher KE, Xu Y, Smith JL: The Dictyostelium kinome - analysis of the protein kinases from a simple model organism. PLoS Genet 2006, 2(3):e38.

19. Kastner PM, Schleicher M, Müller-Taubenberger A: The NDR family kinase $\mathrm{NdrA}$ of Dictyostelium localizes to the centrosome and is required for efficient phagocytosis. Traffic 2011, 12(3):301-312.

20. Pearce $L R$, Komander D, Alessi DR: The nuts and bolts of AGC protein kinases. Nat Rev Mol Cell Biol 2010, 11(1):9-22.

21. Wohlgemuth S, Kiel C, Kramer A, Serrano L, Wittinghofer F, Herrmann C Recognizing and defining true Ras binding domains I: biochemical analysis. J Mol Biol 2005, 348(3):741-758.

22. McPherson JP, Tamblyn L, Elia A, Migon E, Shehabeldin A, Matysiak-Zablocki E, Lemmers B, Salmena L, Hakem A, Fish J, Kassam F, Squire J, Bruneau BG, Hande MP, Hakem R: Lats2/Kpm is required for embryonic development, proliferation control and genomic integrity. EMBO J 2004, 23(18):3677-3688

23. Yabuta N, Okada N, Ito A, Hosomi T, Nishihara S, Sasayama Y, Fujimori A, Okuzaki D, Zhao H, Ikawa M, Okabe M, Nojima H: Lats2 is an essential mitotic regulator required for the coordination of cell division. J Biol Chem 2007, 282(26):19259-19271.

24. Hergovich A, Lamla S, Nigg EA, Hemmings BA: Centrosome-associated NDR kinase regulates centrosome duplication. Mol Cell 2007, 25(4):625-634

25. Yang X, Yu K, Hao Y, Li DM, Stewart R, Insogna KL, Xu T: LATS1 tumour suppressor affects cytokinesis by inhibiting LIMK1. Nat Cell Biol 2004, 6(7):609-617.

26. Li Y, Pei J, Xia H, Ke H, Wang H, Tao W: Lats2, a putative tumor suppressor, inhibits G1/S transition. Oncogene 2003, 22(28):4398-4405.

27. Bothos J, Tuttle RL, Ottey M, Luca FC, Halazonetis TD: Human LATS1 is a mitotic exit network kinase. Cancer Res 2005, 65(15):6568-6575.

28. Yang $X$, Li DM, Chen W, Xu T: Human homologue of Drosophila lats, LATS1, negatively regulate growth by inducing $\mathrm{G}(2) / \mathrm{M}$ arrest or apoptosis. Oncogene 2001, 20(45):6516-6523.

29. lida S, Hirota T, Morisaki T, Marumoto T, Hara T, Kuninaka S, Honda S, Kosai K, Kawasuji M, Pallas DC, Saya H: Tumor suppressor WARTS ensures genomic integrity by regulating both mitotic progression and $\mathrm{G} 1$ tetraploidy checkpoint function. Oncogene 2004, 23(31):5266-5274.

30. Rot G, Parikh A, Curk T, Kuspa A, Shaulsky G, Zupan B: dictyExpress: a Dictyostelium discoideum gene expression database with an explorative data analysis web-based interface. BMC Bioinformatics 2009, 10:265.

31. Gräf R, Daunderer C, Schliwa M: Dictyostelium DdCP224 is a microtubule-associated protein and a permanent centrosomal resident involved in centrosome duplication. J Cell Sci 2000, 113(Pt 10):1747-1758.

32. Reinders $Y$, Schulz I, Gräf R, Sickmann A: Identification of novel centrosomal proteins in Dictyostelium discoideum by comparative proteomic approaches. J Proteome Res 2006, 5(3):589-598. 
33. Clarke M, Engel U, Giorgione J, Müller-Taubenberger A, Prassler J, Veltman D, Gerisch G: Curvature recognition and force generation in phagocytosis. BMC Biol 2010, 8:154.

34. de Rooij J, Bos JL: Minimal Ras-binding domain of Raf1 can be used as an activation-specific probe for Ras. Oncogene 1997, 14(5):623-625.

35. Sutherland BW, Spiegelman GB, Weeks G: A Ras subfamily GTPase shows cell cycle-dependent nuclear localization. EMBO Rep 2001, 2(11):1024-1028.

36. Tuxworth Rl, Cheetham JL, Machesky LM, Spiegelmann GB, Weeks G, Insall RH: Dictyostelium RasG is required for normal motility and cytokinesis, but not growth. J Cell Biol 1997, 138(3):605-614.

37. Mondal S, Bakthavatsalam D, Steimle P, Gassen B, Rivero F, Noegel AA: Linking Ras to myosin function: RasGEF Q, a Dictyostelium exchange factor for RasB, affects myosin II functions. J Cell Biol 2008, 181(5):747-760

38. Kang R, Kae H, Ip H, Spiegelman GB, Weeks G: Evidence for a role for the Dictyostelium Rap1 in cell viability and the response to osmotic stress. J Cell Sci 2002, 115(Pt 18):3675-3682.

39. Bolourani P, Spiegelman G, Weeks G: Determinants of RasC specificity during Dictyostelium aggregation. J Biol Chem 2010, 285(53):41374-41379.

40. Bolourani P, Spiegelman G, Weeks G: Ras proteins have multiple functions in vegetative cells of Dictyostelium. Eukaryotic Cell 2010, 9(11):1728-1733.

41. Hoeller O, Bolourani P, Clark J, Stephens LR, Hawkins PT, Weiner OD, Weeks G, Kay RR: Two distinct functions for Pl3-kinases in macropinocytosis. J Cell Sci 2013, 126(Pt 18):4296-4307.

42. Das M, Wiley DJ, Chen X, Shah K, Verde F: The conserved NDR kinase Orb6 controls polarized cell growth by spatial regulation of the small GTPase Cdc42. Curr Biol 2009, 19(15):1314-1319.

43. Wehland J, Willingham MC: A rat monoclonal antibody reacting specifically with the tyrosylated form of alpha-tubulin. II. Effects on cell movement, organization of microtubules, and intermediate filaments, and arrangement of Golgi elements. J Cell Biol 1983, 97(5 Pt 1):1476-1490.

doi:10.1186/1471-2121-15-25

Cite this article as: Müller-Taubenberger et al:: Regulation of a LATS-homolog by Ras GTPases is important for the control of cell division. BMC Cell Biology 2014 15:25.

\section{Submit your next manuscript to BioMed Central and take full advantage of:}

- Convenient online submission

- Thorough peer review

- No space constraints or color figure charges

- Immediate publication on acceptance

- Inclusion in PubMed, CAS, Scopus and Google Scholar

- Research which is freely available for redistribution 